This is the peer reviewed version of the following article:

Avci C., Ariñez-Soriano J., Carné-Sánchez A., Guillerm V., Carbonell C., I maz I., Maspoch D.. Post-Synthetic Anisotropic Wet-Chemical Etching of Colloidal Sodalite ZIF Crystals. Angewandte Chemie - International Edition, (2015). 54. : 14417 - . 10.1002/anie.201507588,

which has been published in final form at https://dx.doi.org/10.1002/anie.201507588. This article may be used for non-commercial purposes in accordance with Wiley Terms and Conditions for Use of Self-Archived Versions. 


\section{Post-synthetic Anisotropic Wet-Chemical Etching of Colloidal Sodalite ZIF Crystals}

Civan Avci, ${ }^{\dagger, s}$ Javier Ariñez-Soriano, ${ }^{\dagger, s}$ Arnau Carné-Sánchez, ${ }^{\dagger}$ Vincent Guillerm, ${ }^{\dagger}$ Carlos Carbonell, ${ }^{\dagger}$ Inhar Imaz ${ }^{\dagger, *}$ and Daniel Maspoch ${ }^{\dagger,,^{*}}$

\begin{abstract}
Controlling the shape of metal-organic framework (MOF) crystals is important for understanding their crystallization and usefu for myriad applications. However, despite the many advances in shaping of inorganic nanoparticles, post-synthetic shape control of MOFs and, in general, molecular crystals remains embryonic. Herein we report using a simple wet-chemistry process at room temperature to control the anisotropic etching of colloidal ZIF-8 and ZIF-67 crystals. Our work enables uniform reshaping of these porous materials into unprecedented morphologies, including cubic and tetrahedral crystals, and even hollow boxes, via acid-base reaction and subsequent sequestration of leached metal ions. Etching tests on these ZIFs reveal that etching occurs preferentially in the crystallographic directions richer in metal-ligand bonds; that, among these directions, the etching rate tends to be faster on the crysta surfaces of higher dimensionality; and that the etching can be modulated by adjusting the $\mathrm{pH}$ of the etchant solution.
\end{abstract}

Chemical etching is an ancient fabrication method that was used by metal and glass craftsmen to obtain sophisticated surface designs. With the advent of controlling the etching orientation a the microscale and nanoscale, anisotropic wet-chemical etching has become highly utile-useful for shaping many materials for diverse applications..$^{[1,2]}$ For example, the anisotropic we chemical etching of single-crystal silicon in the presence of base is essential in microelectronics manufacturing. ${ }^{[1]}$ Anisotropic etching can also be applied to preparation of meta nanocrystals from oxidative species and coordination ligands, fo which it enables unprecedented morphologies and complexities, and unique physical properties. ${ }^{[2]}$ Here, we introduce the concept of anisotropic wet-chemical etching for metal-organic frameworks (MOFs)-specifically, for the zeolitic-imidazolate framework (ZIF) subfamily.

MOFs (and by extension, ZIFs) are an emerging class of porous materials that show extremely large surface areas $\left(S_{B E T}\right)$ and potential for myriad applications, including gas sorption and separation, catalysis, sensing and biomedicine, among others. ${ }^{[3,4]}$ MOFs are built up from metal ions/clusters connected through organic linkers. Their exposed crystal facets, edges and vertices can exhibit different chemical composition. We hypothesized that agents capable of breaking the coordination bonds between the metal ions/clusters and the organic linkers

[*] C. Avci, J. Ariñez-Soriano, Dr. A. Carné-Sánchez, Dr. V. Guillerm Dr. C. Carbonell, Dr. I. Imaz, Prof. D. Maspoch

ICN2 - Institut Catala de Nanociencia i Nanotecnologia

Campus UAB, 08193 Bellaterra (Barcelona), Spain.

E-mail: inhar.imaz@icn.cat, daniel.maspoch@icn.cat

Prof. D. Maspoch

Institució Catalana de Recerca i Estudis Avançats (ICREA)

08100 Barcelona (Spain).

Supporting information for this article is available on the WWW under $\mathrm{x}$. could be exploited to preferentially etch specific external crystal surfaces (with more density of coordination bonds) over others. We envisioned that such control would enable us to postsynthetically tailor the shape of MOF crystals. To date, postsynthetic random etching of MOF crystals using $\mathrm{H}^{+}, \mathrm{Na}^{+}$and quinone has already been demonstrated. ${ }^{[5,6]}$ Inspired by similar results with zeolites, this strategy has enabled researchers to prepare hierarchical MOF crystals and/or create macropores on the MOF crystal surfaces. ${ }^{[7,8]}$ However, to date, no one has demonstrated the ability to rationally and post-synthetically control the anisotropic etching of a porous MOF crystal in function of its crystal planes, to shape it into different morphologies.

Isostructural ZIF-8 and ZIF-67 are among the most important porous MOF materials known today. They are isomorphous with zeolites, and their 3D frameworks, built up from connecting $\mathrm{Zn}(\mathrm{II})$ ions (ZIF-8) or $\mathrm{Co}(\mathrm{II})$ ions (ZIF-67) through 2-methylimidazole (2-MIM) linkers, show a sodalite topology featuring large cavities (11.6 ^) and small apertures (3.4 A) (see Figure S1) ${ }^{[9]}$ Recently, several studies have shown that the crystal growth of ZIF-8 starts with formation of cubes exposing six $\{100\}$ facets, which gradually evolve into truncated rhombic dodecahedra exposing six $\{100\}$ and twelve $\{110\}$ facets, and finally, into the thermodynamically more stable rhombic dodecahedra, in which only the twelve $\{110\}$ facets are exposed (Figure 1a). ${ }^{[10,11]}$ Figure 1a illustrates the different exposed crystallographic planes for the cubes and for the truncated and non-truncated rhombic dodecahedra. As shown in this figure, each crystal shape has different exposed crystallographic planes, and each crystallographic plane, a distinct chemical composition. In ZIF-8, $\{100\}$ and $\{211\}$ planes contain several $\mathrm{Zn}-2-\mathrm{MIM}$ linkages, whereas the $\{110\}$ and $\{111\}$ planes do not contain any of these linkages (Figure 1b).

By exploiting the aforementioned differences, we devised a method for anisotropic wet etching of colloidal crystals of isostructural ZIF-8 and ZIF-67. Our approach combines a simple acid-base reaction with metal-ion sequestration, which occurs when mixing a colloidal aqueous solution $(2.5 \mathrm{ml})$ of $\mathrm{ZIF}-8$ or ZIF-67 crystals ( $25 \mathrm{mg} ; \mathrm{pH}_{\mathrm{ZIF}-8}=9.2 ; \mathrm{pH}_{\mathrm{ZIF}-67}=10.5$ ) into an aqueous solution $(2.5 \mathrm{ml})$ containing the weak acid xylenol orange $(\mathrm{XO} ; 40 \mathrm{mg})$, whose $\mathrm{pH}$ is adjusted using $\mathrm{HCl}$ or $\mathrm{NaOH}$. When the two solutions are mixed, the 2-MIM linkers become protonated. This in turn breaks the $\mathrm{Zn} / \mathrm{Co}-2-\mathrm{MIM}$ bonds, and slowly and preferentially carves those external crystal surfaces having the highest density of $\mathrm{Zn} / \mathrm{Co}$-2-MIM bonds (i.e carves in the $<100>$ and/or $<211>$ directions). Simultaneously, XO also acts as a chelating agent that coordinates the liberated $\mathrm{Zn}$ (II) and $\mathrm{Co}$ (II) ions to form the well-known, stable and water-soluble $\mathrm{Zn}-\mathrm{XO}$ and Co-XO complexes (e.g. $\mathrm{pKf}_{\mathrm{Zn}-\mathrm{XO}}=6.15$ ), respectively. ${ }^{[12]}$ This reaction sequesters the metal ions, thereby preventing any further (undesired) reaction. 
Figure 1. a) Schematic illustration of the change in crystal morphology during the crystal growth of ZIF-8. Note the change from cube to truncated rhombic dodecahedron to rhombic dodecahedron. The main exposed crystallographic planes are given for all crystal morphologies. $\{100\},\{211\},\{110\}$ and $\{111\}$ a)

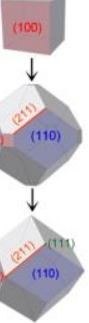

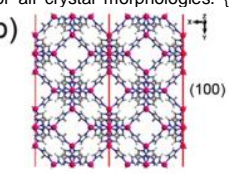
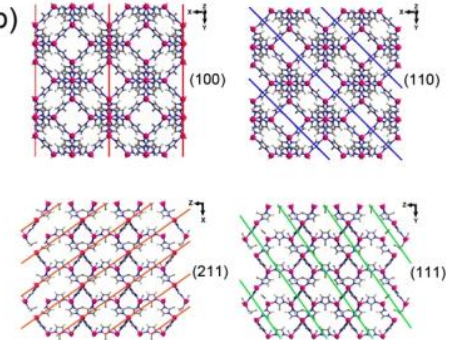

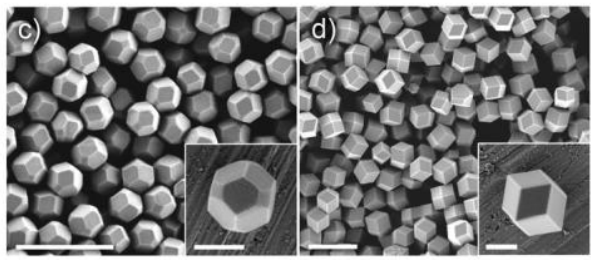

crystallographic planes are represented in red, orange, blue and green, respectively, throughout the manuscript. b) Representations of the crysta structure planes highighted. Zinc ions are represented as pink spheres, and carbon, nitrogen and hydrogen atoms as grey, blue and white slicks, respectively. c, $d$ ) Representative FE-SEM images showing general views of the synthesized truncated (c) and non-truncated (d) rhombic dodecahedra ZIF-8 crystals, and insets).

A first example of our method is shown in Figure 2, which illustrates the sequential, anisotropic, wet-chemical etching of truncated rhombic dodecahedral ZIF-8 crystals into well-defined cubes. To follow the morphologic evolution, colloidal solutions of ZIF-8 crystals (size $=605 \pm 43 \mathrm{~nm}$; Figure 1c) were systematically mixed with $\mathrm{XO}$ solutions that vary in $\mathrm{pH}(8.2,8.0$, 6.0 and 3.0). These mixtures, whose initial $\mathrm{pHs}$ changed to 8.3 , $8.1,6.8$ and 5.8 , respectively, were stirred at $300 \mathrm{rpm}$ for $18 \mathrm{~h}$. We quickly observed two trends from these initial incubations. Firstly, over time, each mixture evolved towards a higher $\mathrm{pH}$ (see Figure S4), suggesting that an acid-base reaction had occurred in each one. Secondly, the initial orange mixtures turned violet, confirming the liberation of $\mathrm{Zn}$ (II) ions and thei subsequent complexation with XO (see Figure S4). After $18 \mathrm{~h}$, the resulting products were analyzed through field-emission scanning electron microscopy (FE-SEM), which indicated that they comprised crystals with the $6\{100\}$ facets preferentially etched over the $12\{110\}$ facets, and that the degree of etching increased with decreasing $\mathrm{pH}$ of the $\mathrm{XO}$ solution (Figure 2a). Consequently, when the $\mathrm{XO}$ solution at $\mathrm{pH}=3$ was used, the areas of each one of the 6 etched $\{100\}$ facets gradually increased until they merged to form well-defined cubes (size $322 \pm 40 \mathrm{~nm}$ ) that exhibited only $\{100\}$ facets (Figure $2 \mathrm{~b}$ ). This etching process clearly indicated a reversal of the reaction that had been observed during the original crystallization process described by Wiebcke et al., ${ }^{[11]}$ whereby $\{100\}$-bounded-faced cubes grow to become $\{100\}$ - and $\{110\}$-bounded faced truncated rhombic dodecahedra (Figure 1a). During this evolution, we also noticed the fingerprints of a slight etching in the $\{211\}$ edges (see Figure S5). Our observations demonstrated that the surface-selective etching is connected to exposure of the metal clusters on the different external surfaces of the ZIF-8 crystal. Thus, the presence of $\mathrm{Zn} / \mathrm{Co}-2-\mathrm{MIM}$ linkages in $\{100\}$ facets and $\{211\}$ edges compared to $\{110\}$ facets is correlated to the higher etching rate in the $\langle 100\rangle$ and the $\langle 211\rangle$ directions relative to the $\langle 110\rangle$ directions. Furthermore, the relatively greater exposed 2-D area of the $\{100\}$ facets compared to that of the 1-D $\{211\}$ edges enabled the etching to proceed more rapidly in the $\langle 100\rangle$ directions, ultimately affording the $\{100\}$-bounded-faced cubes.

Importantly, we were able to apply the same etching process in ZIF- 67 crystals, to morph them from truncated rhombic dodecahedra (size $=628 \pm 66 \mathrm{~nm}$; see Figure S3) into cubes (size $=415 \pm 47 \mathrm{~nm}$; Figure 2c). In this system, the optimum etching conditions were incubation of the ZIF-67 crystals with a XO solution at $\mathrm{pH}$ of 8.0 for $3 \mathrm{~h}$. Also important to highlight is that, for both ZIFs, the simulated (derived from the single crystal structure) and experimental (from cubic crystals) X-ray powder diffraction (XRPD) patterns were consistent (see Figure S6), confirming that the etched cubic crystals were structurally identical to the starting (truncated rhombic dodecahedral) ZIF-8 and ZIF-67 crystals, and that the etching had not affected the initial crystalline integrity of the ZIFs.

Figure 2. a) Schematic illustration and corresponding FE-SEM images (ZIF-8)

a)
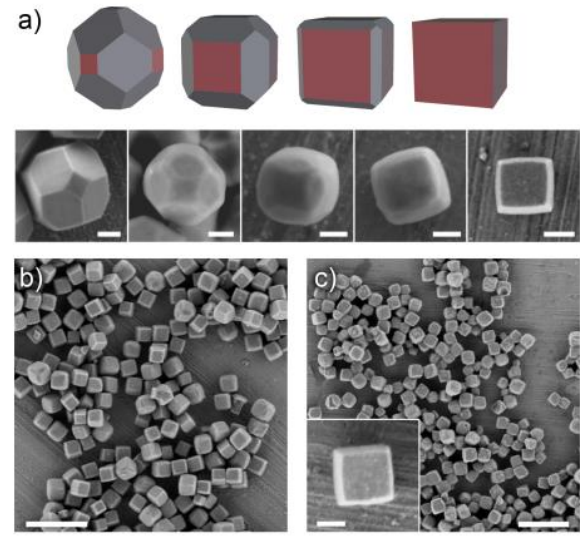

of the change in crystal morphology during the etching of truncated rhombic dodecahedral ZIF-8 and ZIF-67 crystals. Note the change in each case, from truncated rhombic dodecahedron to cube. In each case, etching occurs preferentially in the $\langle 100\rangle$ direction. b,c) Representative FE-SEM images showing general views of the ZIF-8 (b) and ZIF-67 (c) cubes. (Inset) Representative FE-SEM image of a single ZIF-67 cube. Scale bars: $1 \mu \mathrm{m}(\mathrm{b}, \mathrm{c})$
and $100 \mathrm{~nm}$ (a, c-inset). 


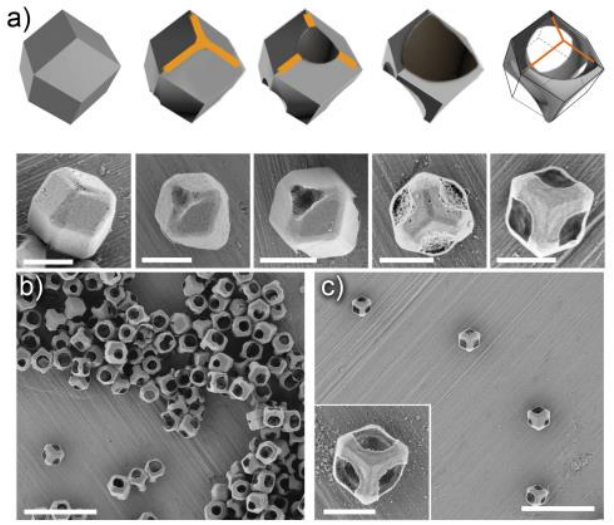

Figure 3. a) Schematic illustration and corresponding FE-SEM images (ZIF67) of the change in crystal morphology during the etching of rhombic dodecahedral ZIF-8 and ZIF-67 crystals, using an etchant solution at pH 2.5 . Note the change from rhombic dodecahedron to hollow box. Etching starts preferentially in the $<211>$ directions. b,c) Representative FE-SEM image FE-SEM image of a single ZIF-67 microbox Scale bars: $5 \mu \mathrm{m}$ (b,c) and 1 m (a, c-inset).

We sought to explore the role of $\mathrm{XO}$ as a chelating agent in the etching process beyond its importance as a proton source. To this end, we performed the ZIF-8 etching experiment without $\mathrm{XO}$, using only $\mathrm{HCl}$ as the etchant solution, to which the crystals at $\mathrm{pH} 5.8$ were exposed for $18 \mathrm{~h}$. Close examination of the product revealed formation of etched ZIF-8 cubes with rougher surfaces than those in the corresponding experiment with $\mathrm{XO}$, as well as a new, unknown crystalline phase that appeared as larger star-like crystals and was subsequently confirmed by XRPD (see Figure S7). These observations evidenced that XO can coordinate with $\mathrm{Zn}(\mathrm{II})$ ions released from the etching of ZIF8 crystals, thereby preventing the precipitation of new solids that would result from the reaction of leached $\mathrm{Zn}$ (II) ions and 2-MIM.

By varying the synthetic conditions of ZIF-8/ZIF-67, we were not only able to construct pure, truncated rhombic dodecahedral crystals, but also obtain perfect rhombic dodecahedral crystals, in which the preferentially etched $\{110\}$ facets evolved into vertices. Under these new conditions, we expected that the etching would be different and thus, would yield distinct ZIF-8 and ZIF-67 crystal morphologies. To study the products formed, we conducted wet-chemical etching experiments similar to those described above, starting from these rhombic dodecahedral crystals (ZIF-8 size $=1.0 \pm 0.1 \mu \mathrm{m}$ and $\mathrm{ZIF}-67$ size $=1.7 \pm 0.3 \mu \mathrm{m}$; Figure $1 \mathrm{~d}$ and see Figure $\mathrm{S} 3$ ). In a first experiment, a colloidal solution of rhombic dodecahedra ZIF-8 (or ZIF-67) crystals was mixed with a $\mathrm{XO} / \mathrm{HCl}$ etchan solution $(\mathrm{pH} \mathrm{2.5)}$, and the resulting mixture was stirred at 300 rpm for $6 \mathrm{~h}$ (ZIF-8) or $3 \mathrm{~h}$ (ZIF-67). Here, chemical etching yielded hollow ZIF-8 and ZIF-67 microboxes with four openings and thin walls (thickness $\approx 40 \mathrm{~nm}$; Figure $3 \mathrm{~b}, \mathrm{c}$ and see Figure $\mathrm{S} 8$ ), as confirmed by FE-SEM and XRPD (see Figure S6). As expected from the etching mechanism (Figure 3a), the edgelength of the resulting boxes was in the range of that of the corresponding initial crystals.

Closer examination of the microboxes described above revealed that they had been formed via anisotropic etching of the original rhombic dodecahedral crystals, from four specific locations. When the process begins, rhombic dodecahedral crystals are preferentially etched from $12\{211\}$ edges that define four equivalent threefold-vertices. As these etched uncovered areas become larger, etching at them becomes progressively faster, leading to formation of pinholes at these vertices, from which the ZIF-8/ZIF-67 are emptied. Note here that although a rhombic dodecahedron has eight threefold-vertices, only half of them are etched, due to the symmetric non-equivalency of the planes. We attributed this phenomenon to the crystallographic polarity ${ }^{[13]}$ of the non-centrosymmetric ZIF-8/ZIF-67, which determines differences in the chemical behavior of the opposite planes $(h k l)$ and $(\bar{h} \bar{k} l)$. These differences could explain etching of half of the $24\{211\}$ edges. Interestingly, the same effect was observed by Anderson et al. in the growing mechanism of $\mathrm{Zn}_{3}\left(\mathrm{PO}_{4}\right)_{2}$ sodalite crystals, ${ }^{[14]}$ which share the same $\bar{I} \overline{4} 3 \mathrm{~m}$ space group as ZIF-8 and ZIF-67.

Formation of these boxes can be explained by the faster etching of the 1-D $\{211\}$ edges compared to the $0-D\{100\}$ vertices in the rhombic dodecahedral crystals. However increasing the $\mathrm{pH}$ of the etchant solution (to 3.5 for $\mathrm{ZIF}-8$ and to 3.25 for ZIF-67) decreased the etching rate in the <211> directions, such that simultaneous etching in the $<211>$ and the $\langle 100\rangle$ directions came to dictate the resulting crystal morphology (Figure 4a). Consequently, etching of rhombic dodecahedra crystals for $18 \mathrm{~h}$ (ZIF-8) or $3 \mathrm{~h}$ (ZIF-67) afforded concave tetrahedra (ZIF- 8 size $=614 \pm 50 \mathrm{~nm}$; and ZIF- 67 size $=$ $745 \pm 83 \mathrm{~nm}$; Figure 4b,c and see Figure S9). Note here that, in the case of ZIF-67, an additional etching step using an etchant solution at $\mathrm{pH}=5.8$ for $30 \mathrm{~min}$ was needed to obtain uniform tetrahedra. These tetrahedra are defined by four $\{111\}$ facets and six $\{100\}$ edges. Again, the XRPD patterns of the products were consistent with those of the initial ZIF-8 and ZIF-67 crystals, confirming that the etched tetrahedral crystals were also structurally identical to initial rhombic dodecahedral crystals. Based on this experiment, we confirmed that we could access completely different ZIF crystal morphologies starting from the same crystal; we realized that we could tune the wet-chemical anisotropic etching by simply changing the $\mathrm{pH}$ of the etchant solution.

One might expect that the heavy chemical treatment in the etching process would affect the adsorption capabilities of the resulting etched ZIF-8/ZIF-67 crystal morphologies, but we did not observe this effect. Indeed, $\mathrm{N}_{2}$ sorption measurements (taken at $77 \mathrm{~K}$ up to $1 \mathrm{bar}$ ) of all the etched ZIF-8 and ZIF-67 crystals proved that they had consistent sorption capacity (Table 1) as that of the initial crystals (see Figure S10-S20). ${ }^{[9,15]}$ In fact, the newly etched materials exhibited slightly higher porosity-a difference that we attributed to the creation of defects ${ }^{[16]}$ during etching. 
a)
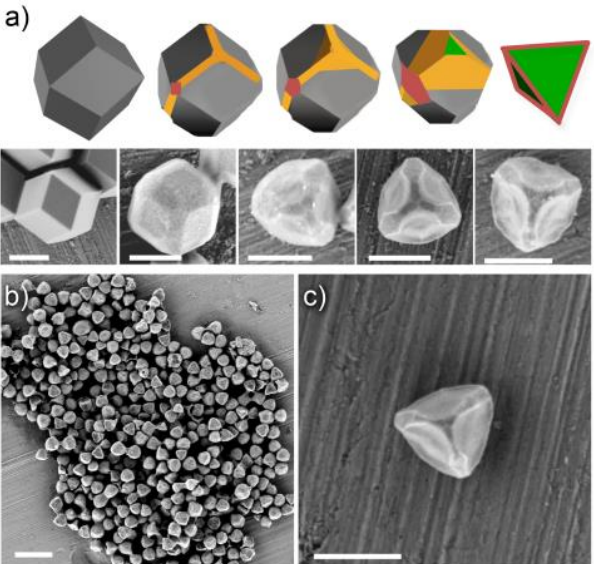

Figure 4. a) Schematic illustration and corresponding FE-SEM images (ZIF-8) of the change in crystal morphology during the etching of rhombic dodecahedral ZIF-8 and ZIF-67 crystals, using an etchant solution at pH 3.5 . Note the change from rhombic dodecahedron to concave tetrahedron. Etching occurs preferentially in the $\langle 211>$ and $<100>$ directions. b) Representative FESEM images showing general views of tetrahedral ZIF-8 crystals. (c) FE-SEM image of a single ZIF-8 tetrahedron. Scale bars: $500 \mathrm{~nm}(\mathrm{a}, \mathrm{c})$ and $2 \mu \mathrm{m}(\mathrm{b})$.

In conclusion, we have demonstrated anisotropic etching of sodalite ZIF crystals. The etching solution in this process is acidified/basified $X O$, which serves to sequentially protonate the imidazolate linkers, break the coordination bonds and sequester the liberated metal ions. We confirmed that etching is surface selective and preferentially favors the metal-ligand bond-rich crystallographic directions and those crystal surfaces of higher dimensionalities. We were able to tune the etching-and consequently, the etched morphologies_by simply adjusting the $\mathrm{pH}$ of the etchant solution, demonstrating that different uniform crystal morphologies (including hollow boxes) can be obtained from the same initial crystal, without any loss in porosity. Inspired by recent advances in the morphologic control inorganic nanocrystals, wWe consider wet-chemical anisotropic etching of MOFs/ZIFs to be an embryonic but promising method for better understanding their crystallization and for postsynthetic, top-down production of these materials in unprecedented shapes that would be inaccessible through conventional chemistry. The access to unique a collection of crystal shapes for a certain MOF should expand their scope of MOFs in numerous practical applications. F-or example. For chromatography, etched morphologies with higher surface-areato-volume ratios with respect to the original shape (e.g. hollow boxes vs. rhombic dodecahedra) may result advantageous for applications in chromatography (e.g. improving the separation of substances) ${ }^{[8]}$ and in heterogeneous catalysis. ${ }^{[17,18]}$ Zhang et at demonstrated that macroporous HKUST-1 microparticles prepared via wet chemical etching gave a faster separation of styrene and ethylbenzene with lower back pressure. (Zhang, J
Mater. Chem. A, 2014, 2, 9085-9090). Also, as already observed in the same tume, some of the now morphologies here reported (o.g. tetrahedra or hollow boxes) present a higher surface-area-to-volume ratio compared to the rhombio dodecahedra, which may result advantageous for applications in heterogeneous catalysis, similar to the results reported by Ryoe et al where MFI zeolite with hierarchical structure exhibited very slow deactivation in catalytic cycles when compared with unmodified MFI zeolite (Ryoo, Chem. Commun., 2006, 4489 4491). Similarly, wet chemical otching of MFI zeolites ${ }^{[19]}$ etched porous morphologies may show has been used also by Wloch et al. to_improve thechanges in the rate of adsorption/desorption rates of this material because of the change in the number of defects present on the crystal surface that can either increase of decrease the rate of adsorption. (W/och, Chem. Mater, 2004, 16 (18), pp 3552-3558). Finally, this novel post-synthetic treatment method could encounter applications in some other fields such as biomedicine, by exploiting the accessible interior of the hollow boxes $[17,18\}$ or in the development-synthesis of -inorganic nanoparticles and hybrid carbons with new morphologies via the well-established calcination approach using MOF crystals as sacrificial templates ${ }^{[1920]}$

Table 1. Summary of the BET surface areas and pore volumes of ZIF-8 and ZIF-67 crystals with different morphologies.

\begin{tabular}{|c|c|c|c|}
\hline MOF & Crystal Morphology & $\begin{array}{l}\text { SBET at first step } \\
\left(m^{2} \cdot g^{-1}\right)\end{array}$ & $\begin{array}{l}\text { Total pore volume } \\
\left(\mathrm{cm}^{3} \cdot \mathrm{g}^{-1}\right) \text { at } \mathrm{P} / \mathrm{P}_{0}= \\
0.95\end{array}$ \\
\hline \multirow[t]{6}{*}{ ZIF-8 } & $\begin{array}{l}\text { Not defined } \\
(\text { polyhedral) })^{[9]}\end{array}$ & 1328 & $\begin{array}{l}0.64^{[a]} \\
(\text { Theo. }=0.54)^{[b]}\end{array}$ \\
\hline & $\begin{array}{l}\text { Rhombic } \\
\text { dodecahedra }\end{array}$ & 1235 & 0.59 \\
\hline & $\begin{array}{l}\text { Truncated rhombic } \\
\text { dodecahedra }\end{array}$ & 1385 & 0.68 \\
\hline & Cubes & 1425 & 0.71 \\
\hline & Boxes & 1310 & 0.69 \\
\hline & Tetrahedra & 1420 & 0.71 \\
\hline \multirow[t]{6}{*}{ ZIF-67 } & Not defined $d^{[15]}$ & 1319 & $\begin{array}{l}0.61 \\
(\text { Theo. }=0.55)^{[b]}\end{array}$ \\
\hline & $\begin{array}{l}\text { Rhombic } \\
\text { dodecahedra }\end{array}$ & 1175 & 0.58 \\
\hline & $\begin{array}{l}\text { Truncated rhombic } \\
\text { dodecahedra }\end{array}$ & 1370 & 0.68 \\
\hline & Cubes & 1540 & 0.80 \\
\hline & Boxes & 1410 & 0.71 \\
\hline & Tetrahedra & 1370 & 0.68 \\
\hline
\end{tabular}

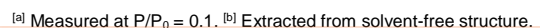

\section{Acknowledgements}

Formatted: Font: Italic, Not Highlight

Formatted: Font: Italic, Not Highlight

Formatted: Font: Italic, Not Highlight

Formatted: Superscript, Not Highlight 
This work was supported by the MINECO-Spain through projects PN MAT2012-30994 and EU FP7 ERC-Co 615954. I.I. and J.A. thanks the MINECO for a RyC and FPU fellowship, respectively, and V.G. is grateful to the Generalitat de Catalunya for a Beatriu de Pinós Fellowship. ICN2 acknowledges the support of the Spanish MINECO through the Severo Ochoa Centers of Excellence Program, under Grant SEV-2013-0295.

Keywords: Metal-Organic Frameworks • Zeolitic-Imidazolate Frameworks $\cdot$ anisotropic etching $\cdot$ hollow particles $\cdot$ porosity

[1] S. Franssila, Introduction to Microfabrication, John Wiley \& Sons, Ltd, Chichester, UK, 2010.

[2] a) E. González, J. Arbiol, V. F. Puntes, Science 2011, 334, 1377-1380; b) A. R. Tao, S. Habas, P. Yang, Small 2008, 4, 310-325; c) Y. G. Sun Y. N. Xia, Science 2002, 298, 2176-2179; d) Y. Yin, R. M. Rioux, C. K Erdonmez, S. Hughes, G. A. Somorjai, A. P. Alivisatos, Science 2004 304, 711-714.

[3] a) Special issue on metal-organic framework materials. Chem. Soc. Rev. 2014, 43, 5415; b) Special issue on metal-organic framework materials. Chem. Soc. Rev. 2009, 38, 1201.

[4] a) P. Horcajada, R. Gref, T. Baati, P. K. Allan, G. Maurin, P. Couvreur, G. Férey, R. E. Morris, C. Serre, Chem. Rev. 2012, 112, 1232-1268; b) M. D. Allendorf, V. Stavila, CrystEngComm., 2015, 17, 229-246; c) N. M. D. Allendorf, V. Stavila, CrystEngComm., 2015, 17, 229-246; c) N.
Stock, S. Biswas, Chem. Rev., 2012, 112, 933-969; d) H. Furukawa, N. Stock, S. Biswas, Chem. Rev., 2012, 112, 933-969; d) H. Furukawa, N.
Ko, Y. B. Go, N. Aratani, S. B. Choi, E. Choi, A. Ö. Yazaydin, R. Q. Snurr, M. O'Keeffe, J. Kim, O. M. Yaghi, Science, 2010, 329, 424-428.

[5] a) Y. Yoo, H.-K. Jeong, Chem. Eng. J. 2012, 181-182, 740-745; b) Hu, S. Furukawa, R. Ohtani, H. Sukegawa, Y. Nemoto J Reboul S. Kitagawa Y Yamauchi Ancew Chem lot Ed 201251 . $984-988$. C) M. Hu, A. Yalk, M. Anga, Y Yamauchi, J.Am, Ohem. Soc. 2013.

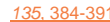
135. 384-39.

[6] G. Wang, Z. Xu, Z Chen, W. Niu, Y. Zhou, J. Guo, L. Tan, Chem Commun. 2013, 49, 6641-6643.

[7] S. El-Hankari, J. Huo, A. Ahmed, H. Zhang, D. Bradshaw, J. Mater. Chem. A. 2014, 2, 13479-13485

[8] A. Ahmed, N. Hodgson, M. Barrow, R. Clowes, C. M. Robertson, A Steiner, P. McKeown, D. Bradshaw, P. Myersa, H. Zhang, J. Mater. Chem. A. 2014, 2, 9085-9090.

[9] a) K. S. Park, Z Ni, A. P. Côté, J. Y. Choi, R. Huang, F. J. Uribe-Romo, H. K. Chae, M. O'Keeffe, O. M. Yaghi, Proc. Natl. Acad. Sci. U. S. A 2006, 103, 10186-10191; b) J.-P. Zhang, Y.-B. Zhang, J.-B. Lin, X.-M. 2006, 103, 10186-10191; b) J.-P. Zhang,
Chen, Chem. Rev. 2011, 112, 1001-1033.

[10] J. Cravillon, R. Nayuk, S. Springer, A. Feldhoff, K. Huber, M. Wiebcke, Chem. Mater. 2011, 23, 2130-2141.

[11] J. Cravillon, C. A. Schröder, H. Bux, A. Rothkirch, J. Caro, M. Wiebcke, CrystEngComm. 2012, 14, 492-498.

[12] a) K. Študlar, I. Janoušek, Talanta 1961, 8, 203-208; b) K. Ogura, S. Kurakami, K. Seneo, J. Inorg. Nucl. Chem. 1981, 43, 1243-1247.

[13] a) P. F. Fewster, P. A. C. Whiffin, J. Appl. Phys. 1983, 54, 4668-4670 b) E. P. Warekois, M. C. Lavine, A. N. Mariano, H. C. Gatos, J. Appl. Phys. 1962, 33, 690-69

[14] M. A. Holden, P. Cubillas, M. P. Attfield, J. T. Gebbie, M. W. Anderson, J. Am. Chem. Soc. 2012, 134, 13066-13073.

[15] Q. Shi, Z. Chen, Z. Song, J. Li, J. Dong, J., Angew. Chem. Int. Ed. 2011, $50,672-675$.

[16] M. J. Katz, Z J. Brown, Y J J Colón, P. W. Siu, K. A. Scheidt, R. Q. Snurr, J. T. Hupp, O. K. Farha, Chem. Commun. 2013, 49, 9449-9451.

117] R. Srivastava. M. Choi, R. Rvoo, Chem. Commun. 2006, 43, 44894491

[18] X. Xu, Z. Zhang. X. Wang, Adv. Mater. 2015, DOl: 10.1002/adma $\underline{201500789 .}$

[19] P. Kortunov, S. Vasenkov, C. Chmelik, J. Kärger, D. M. Ruthven, Wloch. Chem. Mater. 2004, 16, 3552-3358.
(17] a) M. Hu, S. Furukawa, R. Ohtani, H. Sukegawa, Y. Nomoto, J. Robout, S. Kitagawa, Y. Yamauchi, Angew. Chem. Int. Ed. 2012, 51, 984-988;

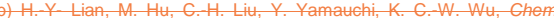
Commun 2012, $48,5151.5153$. C) K. Jayaramulu. K. S. Krishna S. J. Gereg $\mathrm{A}$ Soc. 2013, 135, 384-391

[18] X. XU, Z. Zhang, $X$. Wang, Adv. Mator. 2015, DOl: 10.1002/adma. 201500789

[2019] a) X.-Y. Yu, L. Yu, H. B. Wu, X. W. Lou, Angew. Chem. Int. Ed. 2015, 54, 5331-5335; b) W. Cho, Y. H. Lee, H. J. Lee, M. Oh, Chem. Commun. 2009, 4756-4758; [c] J. Tang, R. R. Salunkhe, J. Liu. N. L. Torad, M. Imura, S. Furukawa, Y. Yamauchi, J. Am. Chem. Soc. 2015 $137,1572-1580 .=$
Formatted: Font: Italic, English (United Kingdom)

Formatted: Font: Italic

Formatted: Font: Bold

Formatted: Font: Italic
Formatted: Font: Italic, English (United Kingdom) Formatted: Font: Bold, English (United Kingdom) Formatted: Font: Italic, English (United Kingdom) Formatted: Font: Italic, English (United Kingdom) Formatted: Font: Italic

Formatted: Font: Bold

Formatted: Font: Italic 
Post-synthetic wet-

chemical anisotropic

etching of colloidal ZIF-8

and ZIF-67 crystals

enables uniform reshaping

of them into

unprecedented shapes,

including cubic and

tetrahedral crystals, and

even hollow boxes, via

acid-base reaction and

subsequent sequestration

of leached metal ions.

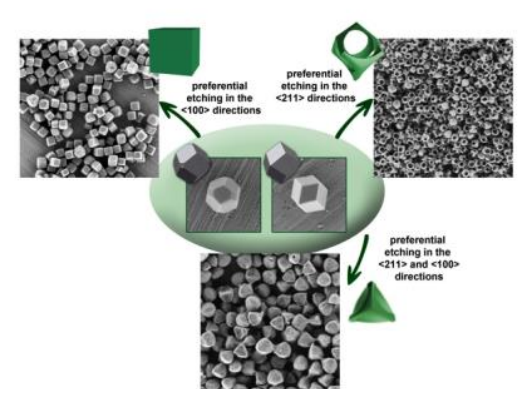

Civan Avci, Javier Ariñez-

Soriano, Arnau Carné-

Sánchez, Vincent Guillerm,

Carlos Carbonell, Inhar

Imaz, ${ }^{*}$ and Daniel

Maspoch*

Page No. - Page No.

Post-synthetic

Anisotropic Wet-

Chemical Etching of

Colloidal Sodalite ZIF

Crystals 